\title{
Convergence et diversité de l'identité professionnelle des enseignantes et des enseignants du secondaire en Communauté française de Belgique
}

\author{
Tensions entre le vrai travail et le sale boulot
}

Convergence and Diversity Related to the Professional Identity

of High School Teachers in Belgium's French Community

Tensions Between the "Real Work " and the "Dirty Work "

\section{Convergencia y diversidad de la identidad profesional de los maestros de secundaria en la Comunidad francesa de Bélgica Tensiones entre el verdadero trabajo y el trajín ingrato}

\section{Branka Cattonar}

Volume 34, numéro 1, printemps 2006

La contribution de l'école au processus de construction identitaire des élèves dans une société pluraliste

URI : https://id.erudit.org/iderudit/1079042ar

DOI : https://doi.org/10.7202/1079042ar

Aller au sommaire du numéro

Éditeur(s)

Association canadienne d'éducation de langue française

ISSN

0849-1089 (imprimé)

1916-8659 (numérique)

Découvrir la revue

Citer cet article

Cattonar, B. (2006). Convergence et diversité de l'identité professionnelle des enseignantes et des enseignants du secondaire en Communauté française de Belgique : tensions entre le vrai travail et le sale boulot. Éducation et francophonie, 34(1), 193-212. https://doi.org/10.7202/1079042ar

\section{Résumé de l'article}

L'objet de cet article est de présenter quelques résultats d'une étude menée sur l'identité professionnelle des enseignantes et des enseignants du secondaire en Communauté française de Belgique. Celle-ci montre que l'identité enseignante est à la fois une construction collective, contextuelle et singulière. L'ensemble des enseignantes et des enseignants paraissent partager un vécu et une conception du métier qui sont en partie communs. En même temps, la manière de vivre et de concevoir le métier varie fortement selon les écoles et l'identité professionnelle semble se construire avant tout à partir des contingences liées aux élèves. Cette construction se fait cependant aussi à partir de l'histoire propre à chaque enseignante ou enseignant, des motivations personnelles et du mode individuel d'adaptation aux conditions de la pratique. En particulier, l'étude met en évidence que l'identité professionnelle des enseignantes et des enseignants est fortement structurée par une tension entre leur conception idéale du " vrai travail » et celle du " sale boulot » qu'ils sont parfois contraints d'exercer, surtout dans les établissements plus difficiles. Cette tension se comprend à la fois par rapport aux histoires personnelles et aux conditions spécifiques de travail, tout en prenant sens par rapport à un contexte éducatif et institutionnel plus large.
Tous droits réservés (C) Association canadienne d'éducation de langue française, 2006
Ce document est protégé par la loi sur le droit d'auteur. L’utilisation des services d’Érudit (y compris la reproduction) est assujettie à sa politique d'utilisation que vous pouvez consulter en ligne. 


\section{Convergence et diversité de l'identité professionnelle des enseignantes et des enseignants du secondaire en Communauté française de Belgique : tensions entre le vrai travail et le sale boulot}

\section{Branka CATTONAR}

Université catholique de Louvain, Louvain-la-Neuve, Belgique

\section{RÉSUMÉ}

L'objet de cet article est de présenter quelques résultats d'une étude menée sur l'identité professionnelle des enseignantes et des enseignants du secondaire en Communauté française de Belgique. Celle-ci montre que l'identité enseignante est à la fois une construction collective, contextuelle et singulière. L'ensemble des enseignantes et des enseignants paraissent partager un vécu et une conception du métier qui sont en partie communs. En même temps, la manière de vivre et de concevoir le métier varie fortement selon les écoles et l'identité professionnelle semble se construire avant tout à partir des contingences liées aux élèves. Cette construction se fait cependant aussi à partir de l'histoire propre à chaque enseignante ou enseignant, des motivations personnelles et du mode individuel d'adaptation aux conditions de la 
pratique. En particulier, l'étude met en évidence que l'identité professionnelle des enseignantes et des enseignants est fortement structurée par une tension entre leur conception idéale du « vrai travail » et celle du « sale boulot » qu'ils sont parfois contraints d'exercer, surtout dans les établissements plus difficiles. Cette tension se comprend à la fois par rapport aux histoires personnelles et aux conditions spécifiques de travail, tout en prenant sens par rapport à un contexte éducatif et institutionnel plus large.

\section{ABSTRACT}

\section{Convergence and Diversity Related to the Professional Identity of High School Teachers in Belgium's French Community. Tensions Between the " Real Work " and the " Dirty Work "}

Branka CATTONAR

Catholic University of Louvain, Louvain-la-Neuve, Belgium

This article presents a few results of a study on the professional identity of high school teachers in Belgium's French community. This study shows that teacher identity is a collective, contextual and singular construction. The teachers seem to have partly common backgrounds and visions of the profession, but how they experience and perceive it varies greatly from school to school, and professional identity seems to be built first and foremost on student-related contingencies.

However, this identity construction also stems from the specific history of each teacher, their personal motivations, and each individual's way of adapting to teaching conditions. In particular, the study shows that the professional identity of teachers is strongly structured by a tension between their ideal concept of the " real work " and the « dirty work » they sometimes have to do, especially in more difficult establishments. This tension can be understood both in relation to the personal stories of each teacher and specific employment conditions, and in terms of a larger educational and institutional context.

\section{RESUMEN}

\section{Convergencia y diversidad de la identidad profesional de los maestros de secundaria en la Comunidad francesa de Bélgica. Tensiones entre el verdadero trabajo y el trajín ingrato}

Branka CATTONAR

Universidad católica de Lovaina, Lovaina-la-Nueva, Bélgica

Este artículo tiene como objetivo presentar algunos de los resultados de un estudio realizado sobre la identidad profesional de los maestros de secundaria de la 
Comunidad francesa de Bélgica. Demuestra que la identidad magisterial es al mismo tiempo una construcción colectiva, contextual y singular. El conjunto de maestros parece compartir una experiencia y una concepción de la profesión parcialmente comunes. Al mismo tiempo, la manera de vivir y de concebir la profesión varia bastante según las escuelas, la identidad profesional parece sobre todo construirse a partir de contingencias relacionadas con los alumnos. Dicha construcción se realiza asimismo a partir de la historia personal de cada maestro, de sus motivos personales, del modo individual de adaptación a las condiciones de la práctica. En particular, el estudio pone en evidencia que la identidad profesional de los maestros está fuertemente estructurada por una tensión entre una concepción ideal del 'verdadero trabajo' y la del 'trajín ingrato' que a veces es necesario realizar, sobre todo en los establecimientos más difíciles. Esta tensión se comprende tanto en relación con las historias personales que con las condiciones laborales específicas, y adquiere sentido en un contexto educativo e institucional más amplio.

\section{Introduction}

Depuis plusieurs années, les enseignantes et les enseignants du secondaire sont confrontés, en Belgique francophone comme dans d'autres pays industrialisés, à de nombreuses évolutions du champ scolaire qui viennent non seulement transformer les conditions d'exercice et la teneur de leur métier, mais aussi bouleverser les bases traditionnelles de leur identité professionnelle : public scolaire plus hétérogène et plus difficilement disposé à entrer dans le jeu scolaire (Dubet, 1991), complexification des finalités éducatives et du rôle enseignant (Tardif et Lessard, 1999), renouvellement rapide des savoirs engendrant une incertitude sur ce qui vaut d'être enseigné (Perrenoud, 1993), remise en cause de la légitimité et de l'autorité des enseignantes et des enseignants les conduisant à devoir se justifier sans cesse (Derouet, 1988), dévalorisation de leur statut social (Chapoulie, 1974; Léger, 1983), désinstitutionnalisation de l'école (Dubet, 2002).

L'identité professionnelle des enseignantes et des enseignants est par ailleurs devenue depuis quelques années un objet social qui structure les discours publics tenus sur leur métier et qui se trouve au centre de réformes impulsées par les autorités scolaires. Dans différents contextes nationaux, on assiste en particulier à une réforme de leur formation qui vise à les "professionnaliser " et à leur faire adopter un nouveau modèle de professionnalité marquant une rupture avec des conceptions plus anciennes de leur travail : les enseignantes et les enseignants n'ont plus à être des «maîtres instruits » accomplissant une "vocation», ni des « techniciens pédagogues " pratiquant un " métier ", ils sont aujourd'hui appelés à devenir des « praticiens réflexifs » exerçant une « profession » (Cattonar et Maroy, 2000; Lang, 
1999; Lessard et Tardif, 2003) ${ }^{1}$. L'identité enseignante est ainsi devenue un enjeu social explicite des politiques scolaires qui la considèrent comme un levier d'amélioration de la qualité, de l'efficacité et de l'équité du système éducatif.

Ces évolutions dans le champ scolaire et le champ professionnel nous ont incités à étudier le métier d'enseignant sous l'angle de l'identité professionnelle parce qu'elle nous a paru constituer un concept sociologique pertinent pour saisir comment s'articulent le vécu subjectif du métier et les conditions sociales de la pratique. Dans cet article, après avoir exposé brièvement notre approche théorique et empirique de l'identité professionnelle, nous allons présenter quelques résultats de deux enquêtes que nous avons réalisées auprès d'enseignantes et d'enseignants du secondaire en Belgique francophone ${ }^{2}$. Celles-ci montrent que l'identité enseignante est une construction fortement liée au contexte de travail (les écoles et leur public), tout en mettant en lumière des tendances collectives (partagées par l'ensemble des enseignantes et des enseignants) et singulières (propres à chaque individu).

\section{Une approche biographique et contextuelle de l'identité professionnelle}

\section{Le cadre d'analyse}

Notre approche sociologique de l'identité professionnelle s'inscrit dans une approche socio-constructiviste (Corcuff, 1995) et l'envisage comme une construction dynamique et continue, individuelle et sociale, résultat plus ou moins stable d'un processus de socialisation biographique et relationnelle, lié au contexte particulier dans lequel il s'inscrit (Cattonar, 2001; Dubar, 1996).

Plus précisément, notre cadre d'analyse énonce trois hypothèses sur la construction identitaire. La première hypothèse pose que l'identité enseignante est une identité spécifique (Blin, 1997) qui est le résultat d'une socialisation professionnelle au cours de laquelle les enseignantes et les enseignants s'approprient activement les normes, règles et valeurs professionnelles propres au groupe. Prenant appui sur des expériences (relativement) communes, comme la formation initiale, cette socialisation est un processus d'identification faisant appel à des «types identitaires " disponibles (Dubar, 1996) : à des modèles idéaux de professionnalité, qui définissent ce que les enseignantes et les enseignants doivent être, faire et savoir idéalement et qui peuvent leur servir de ressources ou de supports identitaires ${ }^{3}$.

La deuxième hypothèse pose que l'identité professionnelle est également une construction singulière, propre à chaque enseignante et enseignant, liée à son histoire personnelle et à ses multiples appartenances sociales, passées et présentes, qu'elles soient familiales, scolaires ou professionnelles (Bourdieu, 1980; Kaufmann, 2001;

1. Le modèle du praticien réflexif met principalement l'accent sur trois nouvelles dimensions du métier : l'adoption d'une démarche réflexive sur la pratique, la mise en œuvre d'une logique d’apprentissage basée sur une pédagogie constructiviste et différenciée, le travail en équipe (Cattonar et Maroy, 2000).

2. Ces deux enquêtes ont été réalisées dans le cadre d'une thèse de doctorat en sociologie (Cattonar, à paraître).

3. Comme exemple de modèle de professionnalité enseignante, on peut citer le modèle traditionnel de l'enseignant « maître instruit » ou celui plus récent du « praticien réflexif ». 
Lahire, 1998). En ce sens, la construction identitaire est un processus biographique continu et l'identité peut être vue comme le résultat d'une transaction entre une identité héritée du passé et une identité visée par l'individu ou imposée par la situation présente (Dubar, 1996).

Enfin, la troisième hypothèse pose que la construction identitaire est aussi un processus relationnel. L'identité est un rapport à soi mais aussi à autrui, d'identification et de différenciation, qui se construit dans l'expérience des relations avec autrui (Sainsaulieu, 1988). Elle peut être vue comme le résultat d'une transaction entre «l'auto-identité » définie par l'individu lui-même et "l'exo-identité » qui lui est attribuée par les personnes avec lesquelles il est en interaction (Berger et Luckmann, 1966; Dubar, 1996; Mucchielli, 1999).

\section{L'approche empirique}

Nous avons réalisé deux études empiriques qui ont visé à la fois à décrire les composantes de l'identité enseignante (les manières de définir et de vivre le métier) et à analyser le processus par lequel elle se construit, en partant des représentations subjectives du métier tout en tenant compte des conditions objectives dans lesquelles se déroulent les expériences professionnelles. Plus précisément, nous avons appréhendé empiriquement l'identité enseignante comme un ensemble de « représentations actives » (cognitives et affectives) structurant les discours tenus sur les pratiques professionnelles (Blin, 1997; Jodelet, 1999). Notre recherche s'est alors attachée à saisir comment l'identité professionnelle s'ancre en même temps dans une histoire personnelle et dans un contexte de travail spécifique et les relations qui y prennent place.

La première étude est une enquête par questionnaire réalisée auprès de 3621 enseignantes et enseignants du secondaire travaillant dans 140 écoles en Communauté française de Belgique ${ }^{4}$. Elle visait à dresser un portrait descriptif de l'identité enseignante à travers plusieurs dimensions (définition du rôle, objectifs pédagogiques valorisés, rapport aux savoirs professionnels, rapport aux différents acteurs impliqués dans l'exercice professionnel, etc.) et à analyser la manière dont le rapport au métier varie selon les caractéristiques socio-individuelles des enseignantes et des enseignants (âge, genre, appartenance sociale, etc.), leurs parcours sociaux (origine sociale, parcours scolaire et professionnel) et le contexte concret d'exercice du métier (les filières d'enseignement, l'établissement scolaire et les élèves) ${ }^{5}$. Dans une logique de "description compréhensive", les données ont été analysées principalement à partir d'outils statistiques comme les tris à plat et les tris croisés (avec l'aide du logiciel SPSS).

4. Le questionnaire (préalablement testé) a été administré à l'ensemble du personnel enseignant travaillant dans les 140 établissements scolaires initialement sélectionnés sur base d'un plan d'échantillonnage stratifié selon les filières d'enseignement.

5. L'enquête par questionnaire visait également à étudier l'évolution de la composition sociale du corps enseignant, les trajectoires d'accès au métier (parcours sociaux, scolaires et professionnels), les conditions d'emploi et de travail des enseignantes et des enseignants ainsi que les différentes dimensions de leur satisfaction professionnelle. Le lecteur intéressé trouvera les principaux résultats de cette enquête dans Cattonar (2002), Maroy et Cattonar (2002) et Maroy (2002). 
Afin de mieux saisir le sens que les enseignantes et les enseignants donnent euxmêmes à leur métier ${ }^{6}$ et étudier la dynamique identitaire dans sa dimension biographique et relationnelle, nous avons également réalisé une enquête par entretien. L'enquête par questionnaire ayant préalablement montré que le rapport au métier variait fortement selon le contexte de travail, pour mieux comprendre cette diversité, nous avons décidé de mener les entretiens dans trois écoles contrastées au niveau du public scolaire ${ }^{7}$. Ce choix se comprend par ailleurs par une situation de forte ségrégation scolaire et sociale qui caractérise le système éducatif en Belgique francophone (Dupriez et Vandenberghe, 2004). Au total, 24 entretiens, semi-directifs et approfondis (d'une durée allant de deux à quatre heures), ont été effectués. Inspirés en partie de la méthode des « récits de vie » (Bertaux, 1997), ils ont abordé le parcours biographique de chaque enseignante et enseignant, en tâchant de retracer l'évolution de la conception du métier à différents moments de la carrière. Par ailleurs, ils ont été réalisés dans une "perspective interactionniste" (Digneffe et Beckers, 1995) cherchant à saisir comment les enseignantes et les enseignants gèrent les «exo-identités " attribuées par autrui (les élèves, les parents, la direction, etc.). Une analyse de contenu à la fois verticale et horizontale (Maroy, 1995) leur a été appliquée ${ }^{8}$. construction qui est en partie commune aux membres du groupe
professionnel. membres du groupe
professionnel.

\section{L'identité} enseignante est une

\section{Une identité collectivement partagée}

\section{Une identité construite essentiellement autour du rapport à l'activité de travail}

Nos deux enquêtes révèlent que l'identité enseignante est une construction qui est en partie commune aux membres du groupe professionnel. Tout d'abord, elles mettent en évidence que la majorité des enseignantes et des enseignants se caractérisent par une identité professionnelle essentiellement construite autour du rapport à l'activité de travail et bien moins autour du rapport à l'emploi ou au statut.

6. L'enquête par entretien visait à saisir la manière dont les enseignantes et les enseignants racontent euxmêmes leur métier et leurs expériences professionnelles par l'analyse de leurs propres " mots identitaires » et « définitions des situations vécues » (Demazière et Dubar, 1997), sans plus partir de catégories prédéfinies comme dans le questionnaire. Les entretiens ont ainsi fait parler le plus librement possible les enseignantes et les enseignants sur la manière dont ils vivent, conçoivent et pratiquent leur métier.

7. Pour contraster les trois écoles, nous les avons sélectionnées selon le retard scolaire moyen et les caractéristiques sociales de leur public scolaire. La première école, qualifiée de " privilégiée », accueille un public scolaire d'origine sociale supérieure et dont le taux de retard scolaire est moins élevé que la moyenne; la deuxième école, qualifiée d'école " moyenne ", accueille un public scolairement et socialement hétérogène, dont le taux de retard scolaire est proche de la moyenne; enfin, la troisième école enquêtée est une école dite " difficile ", qui est en discrimination positive, qui accueille un public scolaire en majorité d'origine immigrée et dont le taux de retard scolaire est plus élevé que la moyenne. Les enseignantes et les enseignants interviewés dans chaque école ont alors été choisis en faisant varier le genre, la filière et le degré d'enseignement. Précisons que la distinction entre école " privilégiée », " moyenne » et " difficile » est perçue et vécue comme telle par les enseignantes et les enseignants des trois établissements, qui utilisent eux-mêmes ces qualificatifs pour décrire leur école.

8. L'analyse verticale (entretien par entretien) s'est attachée à dégager les logiques individuelles en " résumant » le parcours biographique de chaque enseignante et enseignant (en saisissant les " tournants » et « moments charnières ») et à identifier les " phrases banales et récurrentes » indiquant, selon Kaufmann (1996), un marquage social. L'analyse horizontale a consisté à comparer les entretiens entre eux pour faire apparaître les mécanismes sociaux dépassant les particularismes (Demazière et Dubar, 1997). 
Ainsi, dans notre enquête par questionnaire, la plupart déclarent avoir choisi le métier principalement pour le contenu du travail (le travail avec les élèves, l'enseignement de la matière ou la pédagogie) et non pour des conditions d'emploi favorables comme le temps libre ou la sécurité d'emploi.

\section{Tableau 1 : Le rapport initial au métier}

La question posée dans le questionnaire était : « En veillant à vous remettre dans votre état d'esprit de l'époque, pouvez-vous nous dire la principale raison pour laquelle vous avez opté pour le métier d'enseignant? ". Les enseignantes et les enseignants devaient répondre en se positionnant sur une échelle à six positions, allant de " 1 pas important » à " 6 très important » pour chacun des items proposés.

L'importance accordée aux différents aspects du métier au moment du choix

\begin{tabular}{|c|c|c|c|c|c|c|c|}
\hline & \multicolumn{3}{|c|}{ Pas important } & \multicolumn{3}{|c|}{ Très important } & \multirow[b]{2}{*}{ Total (N) } \\
\hline & 1 & 2 & 3 & 4 & 5 & 6 & \\
\hline $\begin{array}{l}\text { Perspective de travailler avec des } \\
\text { jeunes, goût du contact avec les jeunes }\end{array}$ & $2,1 \%$ & $1,8 \%$ & $6,2 \%$ & $13,8 \%$ & $22,4 \%$ & $53,6 \%$ & $100 \%(3337)$ \\
\hline Intérêt pour la matière enseignée & $2,7 \%$ & $2,4 \%$ & $7,0 \%$ & $14,1 \%$ & $23,6 \%$ & $50,2 \%$ & $100 \%(3332)$ \\
\hline $\begin{array}{l}\text { Intérêt pour la pédagogie, donner } \\
\text { aux élèves le goût d'apprendre }\end{array}$ & $3,0 \%$ & $2,1 \%$ & $7,4 \%$ & $14,7 \%$ & $24,4 \%$ & $48,4 \%$ & $100 \%(3329)$ \\
\hline $\begin{array}{l}\text { Assumer une fonction sociale utile, } \\
\text { contribuer à une mission d'intérêt général }\end{array}$ & $8,2 \%$ & $6,4 \%$ & $14,0 \%$ & $20,7 \%$ & $24,3 \%$ & $26,3 \%$ & $100 \%(3315)$ \\
\hline Autonomie dans le travail & $11,6 \%$ & $7,8 \%$ & $16,6 \%$ & $23,5 \%$ & $21,8 \%$ & $18,6 \%$ & $100 \%(3307)$ \\
\hline Compatibilité avec la vie privée & $16,2 \%$ & $7,6 \%$ & $13,7 \%$ & $15,2 \%$ & $15,7 \%$ & $31,7 \%$ & $100 \%(3326)$ \\
\hline Variété dans le contenu du travail & $20,9 \%$ & $11,3 \%$ & $19,4 \%$ & $20,0 \%$ & $14,9 \%$ & $13,5 \%$ & $100 \%(3298)$ \\
\hline Sécurité, stabilité d'emploi & $27,4 \%$ & $12,8 \%$ & $19,3 \%$ & $16,4 \%$ & $12,4 \%$ & $11,8 \%$ & $100 \%(3300)$ \\
\hline Flexibilité dans I'horaire de travail & $34,6 \%$ & $14,3 \%$ & $16,5 \%$ & $15,0 \%$ & $10,1 \%$ & $9,4 \%$ & $100 \%(3302)$ \\
\hline Temps libre, vacances & $30,4 \%$ & $15,3 \%$ & $21,4 \%$ & $14,4 \%$ & $8,9 \%$ & $9,5 \%$ & $100 \%(3315)$ \\
\hline Reconnaissance sociale & $39,4 \%$ & $17,3 \%$ & $19,6 \%$ & $13,6 \%$ & $6,3 \%$ & $3,7 \%$ & $100 \%(3278)$ \\
\hline Régime de pension & $62,8 \%$ & $12,0 \%$ & $12,7 \%$ & $6,1 \%$ & $3,2 \%$ & $3,2 \%$ & $100 \%(3283)$ \\
\hline Salaire & $51,6 \%$ & $18,5 \%$ & $18,7 \%$ & $7,7 \%$ & $2,2 \%$ & $1,3 \%$ & $100 \%(3281)$ \\
\hline Possibilités de carrière & $74,7 \%$ & $11,8 \%$ & $7,2 \%$ & $3,2 \%$ & $1,3 \%$ & $1,8 \%$ & $100 \%(3261)$ \\
\hline
\end{tabular}

Source : Enquête réalisée par le Girsef (UCL), en 1999, auprès de 3621 enseignantes et enseignants du secondaire en Communauté française de Belgique

C'est également au contenu du travail (en particulier, le travail avec les élèves et la matière) qu'ils accordent aujourd'hui le plus d'importance pour leur satisfaction professionnelle. 
Tableau 2 : Le rapport actuel au métier

La question posée dans le questionnaire était : « À quels aspects accordez-vous le plus d'importance? Autrement dit, quels sont les aspects qui importent le plus pour votre satisfaction? ". Les enseignantes et les enseignants devaient répondre en choisissant les aspects qu'ils jugent les plus importants pour leur satisfaction (cinq au maximum).

Les aspects du métier jugés les plus importants pour la satisfaction professionnelle

\begin{tabular}{|c|c|}
\hline & $\begin{array}{c}\text { Pourcentage d'enseignantes } \\
\text { et d'enseignants ayant cité cet aspect } \\
\text { comme étant important }\end{array}$ \\
\hline \multicolumn{2}{|l|}{ Le contenu du travail $(*)$} \\
\hline Le travail et les relations avec vos élèves & $68,7 \%$ \\
\hline L'intérêt pour la matière enseignée & $47,4 \%$ \\
\hline $\begin{array}{l}\text { L'enrichissement personnel, le développement de ses } \\
\text { compétences pédagogiques ou dans la discipline enseignée }\end{array}$ & $33,6 \%$ \\
\hline Assumer une fonction sociale utile & $27,7 \%$ \\
\hline L'intérêt pour la pédagogie & $25,0 \%$ \\
\hline \multicolumn{2}{|l|}{ Les conditions de travail $(*)$} \\
\hline L'autonomie dans le travail & $36,2 \%$ \\
\hline La variété dans le contenu du travail & $13,4 \%$ \\
\hline \multicolumn{2}{|l|}{ Les relations de travail $(*)$} \\
\hline Les relations avec les collègues & $37,7 \%$ \\
\hline Les relations avec le directeur de l'école & $11,3 \%$ \\
\hline \multicolumn{2}{|l|}{ Le niveau ou le type d'enseignement $(*)$} \\
\hline Les niveaux d'étude auxquels ils enseignent & $13,8 \%$ \\
\hline Les filières d'études auxquelles ils enseignent & $9,6 \%$ \\
\hline \multicolumn{2}{|l|}{ Les conditions d'emploi $(*)$} \\
\hline L'équilibre entre vie professionnelle et privée & $45,5 \%$ \\
\hline Le temps libre, les vacances & $30,2 \%$ \\
\hline La flexibilité dans I'horaire de travail & $14,5 \%$ \\
\hline La sécurité d'emploi & $12,0 \%$ \\
\hline Le salaire & $11,9 \%$ \\
\hline Le régime de pension & $3,0 \%$ \\
\hline Les possibilités de carrière et d'avancement & $2,7 \%$ \\
\hline \multicolumn{2}{|l|}{ Le statut social $(*)$} \\
\hline La place des enseignants dans la société & $8,8 \%$ \\
\hline
\end{tabular}

Source : Enquête réalisée par le Girsef (UCL), en 1999, auprès de 3621 enseignantes et enseignants du secondaire en Communauté française de Belgique

(*) Nous avons regroupé les dix-neuf aspects proposés aux enseignantes et aux enseignants en six catégories selon qu'ils concernent plutôt le contenu du travail, l'emploi, les relations de travail, le type d'enseignement et le statut social.

Dans ce rapport au métier centré sur l'activité de travail, les relations avec les élèves occupent une place centrale. Près de 70 \% des enseignantes et des enseignants estiment qu'elles sont primordiales pour leur satisfaction professionnelle et $98 \%$ 
Pratiquement toutes les enseignantes et tous les enseignants considèrent que leur métier consiste avant tout, normalement et idéalement, à donner cours et à enseigner des savoirs aux élèves : c'est, pour eux, leur « vrai travail ». déclarent que les élèves sont les acteurs par lesquels il leur importe le plus d'être reconnus. Notre enquête par entretien montre par ailleurs que la plupart des enseignantes et des enseignants définissent l'enseignement comme un «métier relationnel » qui, par les échanges qu'ils ont avec les élèves, contribue à les « enrichir ». Dans la relation avec les élèves, ce qui semble avant tout compter est le rapport que les élèves ont à leur scolarité. Les enseignants racontent attendre surtout de leurs élèves qu'ils montrent de l'intérêt pour leur cours, qu'ils soient "motivés » et «enthousiastes ». S'ils attendent également qu'ils soient un minimum disciplinés pour pouvoir donner cours, leur principal problème ne constitue cependant pas la violence scolaire tant médiatisée, mais plutôt le désintérêt scolaire ${ }^{9}$. Comme l'a relevé Dubet (1991) pour la France, on pourrait voir dans l'importance accordée au rapport que les élèves ont à leur scolarité l'indice que le public scolaire, ou une partie du moins, n'est pas d'emblée disposé à entrer dans le jeu scolaire et convaincu du sens du travail scolaire tel qu'il est proposé par les enseignantes et les enseignants. Une partie du travail enseignant est alors décrite comme étant consacrée à « motiver » et " convaincre » les élèves, les « intéresser », voire les « séduire " par le maniement de diverses compétences et techniques comme savoir " faire du théâtre », utiliser son humour et son charisme, " contourner la matière » et " raconter des histoires », ou encore mettre en pratique des méthodes pédagogiques participatives. Soutenir, voire créer l'engagement des élèves pour le travail scolaire est présenté comme un véritable travail en soi, de manière variable cependant selon le public scolaire auquel ils ont affaire.

\section{Le " vrai travail " et le " sale boulot "}

Notre enquête par entretien met ensuite en évidence que pratiquement toutes les enseignantes et tous les enseignants considèrent que leur métier consiste avant tout, normalement et idéalement, à donner cours et à enseigner des savoirs aux élèves : c'est, pour eux, leur "vrai travail ». Les autres tâches qu'ils sont amenés à accomplir, de manière variable selon les contextes, comme la socialisation des élèves, l'écoute et la prise en charge de leurs problèmes personnels et sociaux ainsi que la gestion de l'ordre en classe, sont la plupart du temps rejetées comme ne faisant pas normalement partie de leur travail : ils disent ne pas avoir été formés, ne pas être payés, ni être devenus enseignants pour les assurer. Ces tâches sont présentées comme des activités ingrates et peu gratifiantes : elles sont considérées comme du «sale boulot» (Hughes, 1958) qu'ils sont contraints de réaliser, surtout auprès d'élèves « difficiles ${ }^{10}$, pour établir les conditions de travail nécessaires pour pouvoir ensuite travailler sur la matière :

9. Aussi, l'intelligence, le don, le niveau des élèves ou leur réussite scolaire sont rarement mis en avant comme des critères définissant ce qu'est un "bon élève ", peut-être parce que les enseignantes et les enseignants les considèrent comme socialement illégitimes et inavouables.

10. Par "élèves difficiles ", nous entendons les élèves qui ont un taux de retard scolaire plus élevé que la moyenne et qui sont perçus comme tels par les enseignantes et les enseignants. II ne s'agit pas ici de naturaliser le concept de "l'élève difficile ». Nous pensons que celui-ci n'existe pas en dehors du regard porté sur lui par l'enseignante ou l'enseignant. Du point de vue des enseignantes et des enseignants, on pourrait dire que l'élève difficile est surtout celui qui n'est pas assez motivé et pas assez discipliné. Par contre, du point de vue des élèves, on pourrait dire que c'est celui qui narrive pas à trouver des ressources d'identification positive et qui ne perçoit pas ou n'est pas convaincu du sens du travail scolaire tel qu'il leur est proposé. 
« Dans des écoles comme ici, enseigner une matière, c'est important, mais il faut d'abord réconcilier les jeunes avec eux-mêmes, avec le monde dans lequel ils vivent (...). Tant que ça n'est pas résolu, on n'arrive pas à énormément de choses, il n'y a rien à faire. " (Enseignante d'économie, école accueillant un public « difficile»)

«Enseigner, au départ, mon idée, c'était faire passer un savoir. (...) Au départ, j'ai pu l'appliquer véritablement et c'était un vrai plaisir d'enseigner. Et puis petit à petit, on a de plus en plus le rôle d'éducateur. (...) Quand l'éducation prend le pas sur l'enseignement, là ça devient pénible. (...) Normalement, ce n'est pas mon boulot! » (Enseignante d'histoire, école accueillant un public scolairement et socialement hétérogène)

Comme autre rôle assumé, à côté de l'enseignement de la matière, seule l'éducation des élèves au sens large d'apprendre aux élèves des " valeurs humaines " et à « devenir des adultes " semble acceptée. Ainsi, dans notre enquête par questionnaire, la majorité des enseignantes et des enseignants (82\%) ont défini leur rôle comme celui d'un éducateur au sens de « former des êtres humains » et estiment que c'est un rôle «normal » (97\%).

\section{Un relatif malaise collectif}

$\mathrm{Au}$ niveau du vécu, notre enquête par questionnaire montre que le rapport au métier est relativement " mitigé " : si la majorité des enseignantes et des enseignants se déclarent globalement satisfaits de leur situation ( $82 \%$ ), une partie importante se dit néanmoins pessimiste quant à l'avenir (57\%) et cesserait d'enseigner si elle en avait la possibilité ( $41 \%$ partiellement et $18 \%$ totalement). Ce relatif « malaise collectif » se traduit notamment par un large sentiment de dévalorisation sociale de leur métier ${ }^{11}$. Ainsi, $90 \%$ des enseignantes et des enseignants se déclarent insatisfaits de la place de leur métier au sein de la société et $91 \%$ ont le sentiment que leur profession n'est pas bien considérée socialement. Plus précisément, lors des entretiens, beaucoup ont exprimé le sentiment d'être considérés par la "société " comme des « ratés » qui « n'ont pas pu trouver d'autre emploi », qui « gagnent un faible salaire », « travaillent peu et sont tout le temps en vacances », qui exercent un métier " difficile, ennuyeux, répétitif ». Selon les enseignantes et les enseignants, cette image sociale a un impact négatif sur leurs relations avec les élèves dans la mesure où elle vient saper les fondements de leur autorité. En raison de leur dévalorisation sociale, ils pensent que leur autorité n'est pas donnée $a$ priori, mais qu'ils doivent «se battre pour la mériter ", en mettant en œuvre diverses compétences (maîtrise de la matière, séduction, charisme, etc.) et en justifiant sans cesse, voire en négociant les modalités de leur pratique (en particulier, la manière dont ils évaluent les élèves et gèrent la discipline).

11. Ce malaise collectif se manifeste également par une critique systématique de tout ce qui a trait à la place des enseignantes et des enseignants dans le système éducatif, en particulier à l'égard des autorités scolaires par lesquelles ils se sentent fortement déconsidérés. Les enseignantes et les enseignants interviewés ont souvent exprimé le sentiment d'être désappropriés de la définition de leur métier par des autorités « coupées de la réalité » qui leur imposent des réformes dont ils ne voient pas toujours le sens ou qu'ils réprouvent. 


\section{Une identité contextuellement construite}

Si nos deux enquêtes font ressortir une conception et un vécu du métier partagés par une grande partie des enseignantes et des enseignants, elles montrent surtout que la manière de définir et de vivre le métier varie fortement selon le contexte de travail. En ce sens, l'identité enseignante apparaît comme étant, en partie du

La manière de définir et de vivre le métier varie fortement selon le contexte de travail. moins, une construction contextuelle. Elles font apparaître un véritable clivage entre ceux qui travaillent dans des " écoles privilégiées " (où le taux de retard scolaire est plus faible que la moyenne et où les élèves sont décrits positivement comme motivés et disciplinés) et ceux qui enseignent dans des établissements plus " difficiles " (où le taux de retard scolaire est plus élevé ou proche de la moyenne et où les élèves sont perçus comme désintéressés par les cours, indisciplinés ou passifs en classe), au point où l'on peut se demander, à l'instar de Barrère (2000) en France, s'il n'existe pas "deux métiers d'enseignant », définis, vécus et pratiqués différemment selon le public scolaire.

En particulier, notre enquête par entretien ${ }^{12}$ montre que les enseignantes et les enseignants qui travaillent avec de «bons élèves » se déclarent plus souvent satisfaits de leur situation et définissent leur métier essentiellement comme l'enseignement de savoirs, en se centrant sur la formation intellectuelle des élèves. Ceux qui travaillent avec des élèves plus " difficiles » ont par contre davantage tendance à concevoir leur métier comme un travail sur les comportements des élèves : les socialiser, s'occuper de leurs problèmes personnels (sociaux ou psychologiques) et gérer l'ordre en classe. Pour définir leur métier, ces enseignantes et ces enseignants se réfèrent alors très souvent à des catégories sociales ou professionnelles extérieures à l'enseignement auxquelles ils s'identifient, comme les assistants sociaux, les psychologues, les parents ou les policiers. Ces tâches qu'ils exercent, comme nous l'avons déjà souligné, sont considérées dans la plupart des cas comme du "sale boulot " qu'ils sont contraints de réaliser pour pouvoir ensuite enseigner leur matière, objectif qu'ils sont loin d'abandonner. En ce sens, les enseignantes et les enseignants qui travaillent dans un contexte difficile paraissent davantage avoir une conception hétérogène de leur métier. Par ailleurs, ils vivent des insatisfactions professionnelles plus fréquentes et plus intenses. Celles-ci renvoient surtout à une frustration intellectuelle qu'ils ressentent parce qu'ils ne peuvent pas pleinement mettre en pratique leur conception idéale du métier, celle d'apprendre une matière, qu'ils se sont forgée souvent avant de devenir enseignants (parfois dès leur enfance) et qui a présidé à leur choix du métier. Sur la base de l'analyse de la " carrière subjective ${ }^{13}$ des enseignantes et

12. L'enquête par questionnaire montre également que la satisfaction professionnelle et la conception du métier varient selon les caractéristiques des élèves. Ces résultats ont déjà été en partie publiés ailleurs (Cattonar, 2002; Maroy, 2002).

13. Par " carrière subjective », nous entendons les différents moments de l'histoire professionnelle qui sont vécus comme significatifs par les enseignantes et les enseignants dans la constitution de leur identité professionnelle. En reprenant la définition donnée par Goffman, il s'agit de s'attacher aux « aspects moraux » de la carrière, " c'est-à-dire au cycle des modifications qui interviennent dans la personnalité du fait de cette carrière et aux modifications du système de représentations par lesquelles l'individu prend conscience de lui-même et appréhende les autres » (Goffman, 1968). 
Si la manière de vivre et de concevoir le métier se spécifie selon les conditions de travail, elle se diversifie aussi au sein des établissements scolaires selon les enseignants. des enseignants, l'hypothèse que nous faisons est que la plupart ont été socialisés et formés à une conception du métier qui l'envisage essentiellement comme un travail individuel d'apprentissage d'une matière à une classe d'élèves dans laquelle règne un ordre scolaire. Face à des élèves plus « difficiles » (ou simplement non conformes à l'idéal qu'ils en avaient), la mise en œuvre de cette conception du métier devient alors problématique tout en continuant à rester légitime et idéale pour la plupart. Non seulement ils se plaignent fortement d'être contraints à exercer des tâches qu'ils considèrent comme du " sale boulot » au détriment de leur " vrai travail », mais l'enseignement de la matière lui-même leur apporte peu de satisfaction dans la mesure où il est perçu comme fort réduit, comme se limitant à apprendre des « savoir-faire " aux élèves, voire des « choses utiles » :

«Ici, je ne suis pas du tout un prof contenu au sens de savoir. (...) Par contre, je pense qu'il y a énormément d'apprentissages en termes de savoir-faire. Apprendre à lire un texte, apprendre à faire un résumé... Il y a des apprentissages, mais pour moi, ce ne sont pas des savoirs." (Enseignante de français, école accueillant un public « difficile»)

La situation n'est cependant pas totalement tranchée. Les enseignantes et les enseignants qui travaillent avec un public scolaire plus « difficile» peuvent également ressentir de grandes satisfactions, en particulier liées aux relations avec les élèves décrites comme plus chaleureuses et à un sentiment d'utilité sociale lorsque « ils y arrivent » (à faire travailler les élèves ou à les " accrocher »). Par ailleurs, ceux qui travaillent dans des contextes plus favorables peuvent aussi ressentir des insatisfactions professionnelles. La relation avec les élèves y est notamment vécue comme moins gratifiante et les enseignantes et les enseignants ressentent parfois du mépris social.

\section{Une identité biographique singulière}

Si la manière de vivre et de concevoir le métier se spécifie selon les conditions de travail, elle se diversifie aussi au sein des établissements scolaires selon les enseignantes et les enseignants. Ainsi, tous ceux qui travaillent dans les mêmes conditions ne partagent pas le même vécu, ni la même conception du métier ${ }^{14}$. En particulier, notre enquête par entretien montre que l'identité professionnelle des enseignantes et des enseignants se construit à partir de leur propre histoire, leurs motivations personnelles et leur mode ou capacité "d'adaptation » aux conditions concrètes de la pratique $^{15}$. En ce sens, l'identité professionnelle est également une construction biographique singulière.

14. Les écoles ne doivent donc pas être perçues comme des lieux de consensus et composés d'un corps enseignant qui s'accorderait sur les modalités et les objectifs de son activité. Au contraire, notre enquête par entretien montre qu'au sein des établissements coexistent des manières différentes de concevoir le travail d'enseignant qui peuvent parfois entrer en opposition, en particulier en ce qui concerne le mode de rapport aux élèves, la gestion de la discipline et l'investissement professionnel dans les projets de l'école.

15. Notre enquête par questionnaire montre par ailleurs que le rapport au métier varie selon des caractéristiques socio-individuelles comme le genre ou l'âge (voir Cattonar, 2002). 


\section{Une tension identitaire inscrite dans une histoire professionnelle}

L'hypothèse que nous faisons est que le vécu des enseignantes et des enseignants est fortement structuré par une tension entre un idéal professionnel et les conditions réelles de la pratique : entre le "vrai travail » souhaité et le « sale boulot » contraint. Cette tension ne se comprend pas seulement au regard de leur contexte de travail, mais aussi au regard de la manière dont ils ont investi leur orientation professionnelle, de leur préconception du métier et de leur vision initiale des élèves.

Notre enquête par entretien montre que cette tension prend sens par rapport à une " carrière subjective " qui débute avant même l'entrée dans le métier. C'est en effet durant leur socialisation préprofessionnelle (qui comprend les expériences antérieures à la formation et à l'entrée dans le métier) et en particulier pendant leur propre scolarité au secondaire que se forge une première conception du métier, laquelle semble très souvent perdurer en tant qu'idéal à travers les expériences professionnelles ultérieures. Cette préconception idéale correspond dans la plupart des cas à celle du « vrai travail » (c'est-à-dire transmettre des savoirs en ayant une bonne relation avec les élèves) qu'ils mettaient alors parfois déjà en pratique lors de jeux enfantins pendant lesquels ils « jouaient à faire la classe » ou qu'ils éprouvaient dans des mouvements de jeunesse.

Cette préconception dominante ne semble pas remise en question par la formation initiale, laquelle est rarement vécue comme un moment significatif de leur carrière. La plupart considèrent que leur formation n'a exercé aucune influence sur leur manière de concevoir et de pratiquer le métier ${ }^{16}$. Ils sont en outre fort critiques à son égard et ils lui reprochent notamment de ne pas les avoir suffisamment formés aux compétences relationnelles et psychologiques nécessaires pour remplir les fonctions éducatives qu'ils sont amenés à remplir, c'est-à-dire leur " sale boulot ». Leur formation initiale, selon les dires des enseignantes et des enseignants, les aurait préparés principalement à l'enseignement d'une matière et nous pourrions supposer que, par là, elle légitime et renforce leur préconception du métier et tend à accroître les futures tensions que certains vivront entre leur « vrai travail » et leur « sale boulot».

L'entrée dans le métier constitue le premier moment où les enseignantes et les enseignants vont véritablement confronter leur préconception du métier aux conditions réelles de la pratique. Comme l'ont également montré d'autres études, ce moment constitue souvent une "expérience critique" (Baillauquès, 1999; Nault, 1999) vécue sous le mode d'un " choc de la réalité » (Kelchtermans et Ballet, 2002). En particulier, il va amener certaines enseignantes et certains enseignants à réviser ou élargir leur préconception du métier en y intégrant le " sale boulot » jusque-là ignoré. Ce «choc de la réalité » n'est pas un phénomène propre aux enseignantes et aux enseignants et il peut être décrit comme un " passage à travers le miroir » (Hughes, 1958), au cours duquel tout travailleur débutant fait la découverte de la "réalité désenchantée » du monde professionnel. Dans le cas des enseignantes et des ensei-

16. Cela ne signifie pas que la formation nait aucun effet. Plusieurs études montrent au contraire que la formation constitue un moment important de l'élaboration identitaire (Baillauquès, 1999; Nault, 1999). Notre enquête par questionnaire met par ailleurs en évidence des conceptions différentes du métier selon la formation suivie (voir Cattonar, 2002). 
gnants, il consiste à découvrir que les élèves sont loin de l'image idéale qu'ils s'en faisaient avant (les élèves ne partagent pas leur intérêt pour la matière, ne sont pas motivés à apprendre, ne sont pas « bien éduqués », etc.), que donner cours ne va pas de soi, qu'enseigner implique du « sale boulot». Cette découverte peut se faire plus tardivement dans la carrière, par exemple lors d'un changement d'affectation. C'est ce qu'évoque cette enseignante qui, avant d'arriver dans une école « difficile », a travaillé une quinzaine d'années dans une « bonne école»:

"Ça été une révélation pour moi. (...) Moi, j’avais l'habitude : j'arrive en classe, on commence à donner cours et ça ne posait pas de problème. (...) J'ai vécu une déstabilisation quand je suis arrivée ici. (...) Finalement, c'est se dire : Quoi? Qu'est-ce que c'est ça? On dit quelque chose et on ne le fait plus. Ils répondent, ils sont grossiers, ils ne remettent pas leurs travaux, ils s'en fichent, ils n'ont pas leurs notes de cours. (...) On commence alors à se poser des questions. On se dit : est-ce que c'est moi qui fait que? (...) Il a bien fallu trouver autre chose, sinon ça ne marchait pas. (...) Au départ je mettais plutôt au centre la matière et essayer de la faire aimer. Je crois que ça reste vrai maintenant, mais cela s'est fort élargi à des tas d'autres choses et je me sens actuellement plus style éducateur. » (Enseignante d'économie, école accueillant un public « difficile»)

Cette confrontation avec des élèves « inattendus " peut être décrite comme le passage d'une idéalisation initiale du métier et des élèves à une conception plus " réaliste », qui engage un travail d'ajustement des références passées aux conditions présentes de la pratique et qui parfois s'apparente à un " travail de deuil " (Dubet, 2002) impliquant de revoir ses préconceptions ou de s'en défaire. Beaucoup vont alors "s'installer dans une dualité " (Hughes, 1958) entre leur conception idéale du métier, celle du "vrai travail ", et la réalité de la pratique, celle qui comporte une dose, variable selon les contextes de travail, de "sale boulot». Cette dualité est plus ou moins forte selon le type d'école où ils continuent leur carrière. Elle est plus vivement ressentie dans les établissements plus difficiles où l'écart entre la conception dominante du métier forgée antérieurement autour de l'image de l'élève " normal » et les conditions réelles d'exercice est plus grand, renforçant l'importance de ce que Van Zanten (2001) appelle la "socialisation secondaire en situation " (dans l'établissement) à l'origine de plus grandes « révisions identitaires ».

\section{Une tension diversement vécue}

La dualité entre l'idéal professionnel et les conditions réelles de la pratique est aussi plus ou moins fortement ressentie selon les enseignantes et les enseignants et leurs premières motivations professionnelles et plus ou moins bien vécue selon leur capacité d'adaptation individuelle et leurs ressources personnelles.

Ainsi, face à des élèves "difficiles", seuls certains vivent une forte "tension identitaire " qui s'exprime par une grande insatisfaction professionnelle liée à l'impossibilité de pratiquer le métier comme souhaité et parfois ressentie en termes de "sur-qualification" (Barrère, 2000). Cette tension se rencontre le plus souvent chez 
des enseignantes et des enseignants qui sont venus au métier principalement par " passion pour une matière » et qui ne renoncent pas à leur idéal de départ centré sur la transmission de leur discipline. Leurs attentes initiales (enseigner la matière et s'enrichir soi-même intellectuellement) n'ayant pas été rencontrées, ils expriment une forte déception, parfois même une perte du sens du métier : « on se pose la question du pourquoi encore enseigner », « on se sent tout nus car on nous a dépouillés de la transmission du savoir » disent-ils. On pourrait dire qu'ils se sentent « orphelins de leurs identifications passées », qu'ils sont «blessés dans leurs croyances incorporées " (Dubar, 2000). Ils vivent dans une situation où leur identité n'est pas " confirmée dans les interactions sociales significatives" pour reprendre les termes de Berger et Luckmann (1986). Leur tension identitaire est alors souvent vécue sous le mode d'un "conflit externe" avec d'autres acteurs rendus responsables de leur insatisfaction : avec les élèves décrits comme " difficiles " et " non motivés », avec les parents jugés "démissionnaires ", avec la direction de l'école dont la politique de gestion de la discipline est estimée trop laxiste ou encore avec les réformes scolaires perçues comme faisant passer au second plan l'enseignement de la matière (au profit des compétences et des savoir-faire, par exemple). Cette tension s'apparente aussi parfois à un "conflit interne " car plusieurs ont conscience de faire un "travail socialement utile» tout en se sentant profondément frustrés intellectuellement.

Pour " tenir le coup ", ces enseignantes et ces enseignants peuvent développer différentes "stratégies de survie" (Van Zanten, 2001). Certains arrivent par des " compensations extérieures » à diminuer leur tension identitaire et à trouver ailleurs des satisfactions non comblées par leur travail à l'école, en s'investissant, par exemple, dans des mouvements pédagogiques, des organisations syndicales ou des formations continuées. Ces investissements extérieurs leur permettent de se ressourcer, " d'avoir de l'air ", mais aussi de " prendre du recul ", " d'analyser autrement la difficulté avec leurs élèves" et ainsi de redonner du sens à leur travail. D'autres enseignantes et enseignants adoptent des stratégies de survie relevant d'une " logique de positivisation des élèves " (Van Zanten, 2001), en développant à leurs égards un regard plus compréhensif. Enfin, certains disent s'appuyer sur leur " réserve d'énergie " construite lors d'expériences d'enseignement antérieures et positives.

Face à des élèves " difficiles ", toutes les enseignantes et tous les enseignants ne sont cependant pas insatisfaits de leur métier, tous ne se sentent pas frustrés intellectuellement, tous ne rechignent pas à assurer le « sale boulot ». Certains ne vivent aucune tension identitaire parce qu'ils avaient dès le départ une conception large du métier et ont toujours pensé que les fonctions éducatives faisaient " normalement » partie de leur travail. Il s'agit d'enseignantes et d'enseignants, plus rares, dont le rapport initial au métier ne s'est pas construit entièrement sur un "amour de la matière » mais aussi sur un attachement à l'enseignement en tant que tel. D'autres enseignantes et enseignants vivent moins fortement cette dualité parce qu'ils avaient au départ un rapport faible au métier (sans être pour autant négatif) : venus essentiellement à l'enseignement pour avoir un emploi et après d'autres expériences professionnelles, ils investissent aujourd'hui leur métier en ayant un rapport à la fois 
plus détaché (ils n'expriment aucun attachement affectif au métier, à leur matière et/ou aux élèves) et plus pragmatique («il s'agit de faire son boulot»). Leurs expériences professionnelles antérieures en dehors de l'enseignement sont souvent présentées comme leur permettant de relativiser les difficultés avec leurs élèves.

Enfin, face à des élèves avec lesquels ils ne pouvaient pas mettre en pratique leur conception idéale du métier, certains racontent avoir vécu une « conversion identitaire » : si, au départ, ils concevaient le métier uniquement comme « transmettre des savoirs ", ils disent s'être " adaptés » à leurs élèves et, sans abandonner l'objectif de travailler la matière, ils font désormais aussi de l'éducation et de la socialisation sans que cela ne les « dérange ». Cette adaptation ne s'est toutefois pas faite sans difficultés et ils évoquent une déstabilisation suivie d'une remise en cause de soi. Ces enseignantes et enseignants relativisent alors la place qu'occupe l'enseignement de la matière pour valoriser davantage leur travail d'éducation et de socialisation. Cette conversion identitaire s'accompagne aussi d'un rapport plus compréhensif aux élèves. On pourrait parler d'une " adaptation contextuelle" (Van Zanten, 2001) qui concerne aussi bien les objectifs de travail que les manières de l'exercer (mise en pratique de pédagogies alternatives, contenus des cours rendus plus accessibles et attrayants, etc.).

\section{Conclusion}

Si la manière de vivre et de gérer les difficultés du métier est singulière, il ne faut cependant pas la réduire à une seule question d'adaptation individuelle et de décalage ou de concordance entre un idéal fantasmé et la réalité concrète. Si l'exercice du métier est une épreuve personnelle, la base du vécu professionnel est également sociale. En ce sens, la réalité du monde enseignant ne se réduit pas aux seuls représentations et vécus subjectifs, elle s'inscrit dans des conditions sociales objectives.

Nous pensons que les tensions vécues par plusieurs enseignantes et enseignants prennent sens par rapport aux transformations qu'ont connues ces dernières années les conditions d'exercice de leur métier, en particulier l'évolution du public scolaire consécutive à la massification de l'enseignement secondaire dans les années 1960. Celle-ci est venue saper les bases traditionnelles de leur identité professionnelle et les amène à un travail de reconstruction du sens de leur métier qui ne va pas de soi. Selon Dubet, la massification de l'enseignement a ainsi «complètement déstabilisé un modèle d'enseignement sans que la plupart des acteurs aient pour autant renoncé à ses principes, à ses valeurs et à son imaginaire ". L'expérience de travail du personnel enseignant est alors « dominée par le choc d'une représentation idéale d'un métier avec des conditions de travail qui la rendent impossible à réaliser " (Dubet, 2002). Barrère (2000) parle d'un « deuil intellectuel» (celui de travailler de manière enrichissante sur la matière) que sont amenés à faire aujourd'hui la plupart des enseignantes et des enseignants. 
Si la construction de l'identité professionnelle se réalise à partir de I'histoire personnelle de chaque enseignante et de chaque enseignant et s'enracine dans un contexte spécifique de travail, celui de l'établissement scolaire, elle prend également sens par rapport à un contexte éducatif et institutionnel plus large.
Par ailleurs, toutes les enseignantes et tous les enseignants ne sont pas égaux face à l'évolution du public scolaire, dans la mesure où elle va de pair, en Belgique francophone, avec une ségrégation forte du système scolaire qui relègue à certains établissements et à certains enseignants et enseignantes la gestion des élèves « difficiles ». Cette ségrégation pose la question de l'équité au niveau des membres du personnel enseignant dans la mesure où elle se traduit par une inégalité dans leurs conditions de travail et leur vécu professionnel. Elle pose également question au niveau des élèves dans la mesure où ceux-ci, selon l'établissement où ils font leur scolarité, ont affaire à des enseignantes et à des enseignants qui ont un rapport au métier différencié et qui conçoivent différemment leur travail.

Enfin, il nous semble que le vécu professionnel des enseignantes et des enseignants doit être resitué par rapport à la définition institutionnelle de leur travail. Ainsi, nous pensons que la situation des enseignantes et des enseignants travaillant dans des contextes plus difficiles serait plus aisée s'ils avaient à leur disposition un modèle de professionnalité légitimant et valorisant les fonctions éducatives qu'ils sont amenés à exercer et qu'ils jugent le plus souvent anormales. En effet, en Communauté française de Belgique, les fonctions de socialisation des élèves ou de gestion de l'ordre ne font pas actuellement l'objet d'une " modélisation » et ne sont pas présentées comme des tâches à part entière et valorisées du travail enseignant ${ }^{17}$. Le fait que la définition institutionnelle du rôle des enseignantes et des enseignants et de leurs compétences reste principalement centrée sur l'apprentissage d'une matière, pourrait renforcer l'idée que les autres fonctions ne font pas «normalement " partie de leur travail. En ce sens, on pourrait dire que la construction de l'identité professionnelle de ces enseignantes et enseignants se déroule sans " cadre légitimateur" dans lequel pourrait prendre sens leur expérience professionnelle. Cette absence de modèle d'identification propre n'a pas qu'un effet symbolique. Elle se traduit concrètement par le fait que leur formation professionnelle ne semble pas les y préparer, comme s'en plaignent de nombreux enseignants et enseignantes. Plus encore, on pourrait se demander si leur formation les arme suffisamment à faire face, au niveau pédagogique, aux difficultés rencontrées avec leurs élèves : dans quelle mesure ceux et celles qui travaillent dans des contextes difficiles ne considèrent-ils pas que l'enseignement de la matière est réduit et ne se retournent-ils pas vers des fonctions plus éducatives parce qu'ils se trouvent démunis sur le plan des méthodes pédagogiques?

En définitive, le vécu actuel des enseignantes et des enseignants nous semble s'inscrire dans un contexte éducatif particulier (celui de la massification et de la ségrégation du système scolaire) et un contexte institutionnel qui ne fournit pas à tous un modèle de référence par rapport auquel pourrait prendre sens l'entièreté de leur expérience de travail, ni ne les arme suffisamment pour faire face aux difficultés du métier. Si la construction de l'identité professionnelle se réalise à partir de l'histoire personnelle de chaque enseignante et de chaque enseignant et s'enracine dans

17. À côté de l'apprentissage de la matière, seules la recherche du développement personnel des élèves et leur préparation à devenir des citoyens responsables sont présentées comme des missions officielles par les pouvoirs publics. 
un contexte spécifique de travail, celui de l'établissement scolaire, elle prend également sens par rapport à un contexte éducatif et institutionnel plus large.

\section{Références bibliographiques}

BAILLAUQUES, S. (1999). Ce que l'entrée dans la carrière révèle du rapport des enseignants à la formation : éléments d'une problématique de professionnalisation, dans Jeunes enseignants et insertion professionnelle. Un processus de socialisation? De professionnalisation? De transformation?, sous la direction de J.-C. Hétu, M. Lavoie et S. Baillauquès. Paris, Bruxelles : De Boeck Université, pp. 21-42.

BARRERE, A. (2000). Sociologie du travail enseignant. L'Année sociologique, vol. 50, $\mathrm{n}^{\circ} 1$, pp. 469-492.

BERGER, P., LUCKMANN, T. (1986). La construction sociale de la société. Paris : Méridiens Klincksieck.

BERTAUX, D. (1997). Les récits de vie. Perspectives ethnosociologiques. Paris : Nathan.

BLIN, J.-F. (1997). Représentations, pratiques et identités professionnelles. Paris : L'Harmattan.

BOURDIEU, P. (1980). Le sens pratique. Paris : Les Éditions de Minuit.

CATTONAR, B. (à paraître). L'identité professionnelle des enseignants du secondaire. Approche biographique et contextuelle. Thèse de doctorat. Université catholique de Louvain.

CATTONAR, B. (2002). Homogénéité et diversité des identités professionnelles enseignantes, dans Les écoles d'enseignement secondaire et leurs enseignants, sous la direction de C. Maroy. Bruxelles : De Boeck Université, pp. 171-208.

CATTONAR, B. (2001). Les identités professionnelles enseignantes. Ebauche d'un cadre d'analyse. Les Cahiers de Recherche du Girsef, ${ }^{\circ} 10$.

CATTONAR, B., MAROY, C. (2000). Rhétorique du changement du métier d'enseignant et stratégie de transformation de l'institution scolaire. Éducation et Sociétés. Revue internationale de sociologie de l'éducation, $\mathrm{n}^{\circ} 6$.

CHAPOULIE, J.-M. (1974). Le corps professoral dans la structure de classe. Revue française de Sociologie, XV, pp. 155-200.

CORCUFF, P. (1995). Les nouvelles sociologies. Constructions de la réalité sociale. Paris : Nathan Université.

DEMAZIERE, D., DUBAR, C. (1997). Analyser les entretiens biographiques. L'exemple des récits d'insertion. Paris : Nathan. 
DEROUET, J.-L. (1988). La profession enseignante comme montage composite. Les enseignants face à un système de justification complexe. Éducation permanente, $\mathrm{n}^{\circ}$ 96, pp. 61-71.

DIGNEFFE, F., BECKERS, M. (1995), De l'individuel au social : l'approche biographique, dans Pratiques et méthodes de recherche en sciences sociales, sous la direction de L. Albarello et alii. Paris : Armand Colin, pp. 145-173.

DUBAR, C. (2000). La crise des identités. L'interprétation d'une mutation. Paris : Presses Universitaires de France.

DUBAR, C. (1996). La socialisation. Construction des identités sociales et professionnelles. Paris : Armand Colin.

DUBET, F. (2002). Le déclin de l'institution. Paris : Éditions du Seuil.

DUBET, F. (1991). Les lycéens. Paris : Éditions du Seuil.

DUPRIEZ, V., VANDENBERGHE, V. (2004). L'école en Communauté française de Belgique : de quelle inégalité parlons-nous?. Les Cahiers de recherche en Éducation et Formation, ${ }^{\circ} 20$.

GOFFMAN, E. (1968). Asiles. Etudes sur la condition sociale des malades mentaux et autres reclus. Paris : Les Éditions de Minuit.

HUGHES, E. C. (1958). Men and their work. Glencoe : The Free Press.

JODELET, D. (1999). Représentations sociales : un domaine en expansion, dans Les représentations sociales, sous la direction de D. Jodelet. Paris : Presses Universitaires de France, pp. 47-78.

KAUFMANN, J.-C. (2001). Ego. Pour une sociologie de l'individu. Une autre vision de l'homme et de la construction du sujet. Paris : Nathan.

KAUFMANN, J.-C. (1996). L'entretien compréhensif. Paris : Nathan.

KELCHTERMANS, G., BALLET, K. (2002). The micropolitics of teacher induction. A narrative-biographical study on teacher socialisation. Teaching and Teacher Education, $\mathrm{n}^{\circ} 18$, pp. 105-120.

LAHIRE, B. (1998). L'homme pluriel. Les ressorts de l'action. Paris : Nathan.

LANG, V. (1999). La professionnalisation des enseignants. Sens et enjeux d'une politique institutionnelle. Paris : Presses Universitaires de France.

LEGER, A. (1983). Enseignants du secondaire. Paris : Presses Universitaires de France.

LESSARD, C., TARDIF, M. (2003). Les identités enseignantes. Analyse de facteurs de différenciation du corps enseignant québécois 1960-1990. Sherbrooke : Éditions du CRP.

MAROY, C. (2002). Contexte de travail, climat d'établissement et satisfaction professionnelle des enseignants, dans Les écoles d'enseignement secondaire et leurs enseignants, sous la direction de C. Maroy. Bruxelles : De Boeck Université, pp. 131-169. 
MAROY, C. (1995). L'analyse qualitative d'entretiens, dans Pratiques et méthodes de recherche en sciences sociales, sous la direction de L. Albarello et alii. Paris : Armand Colin, pp. 83-110.

MAROY, C., CATTONAR, B. (2002). L'évolution sociologique des enseignants du secondaire en Belgique francophone, dans Les écoles d'enseignement secondaire et leurs enseignants, sous la direction de C. Maroy. Bruxelles : De Boeck Université, pp. 93-129.

MUCCHIELLI, A. (1999). L'identité. Paris : Presses Universitaires de France.

NAULT, T. (1999). Éclosion d'un moi professionnel personnalisé et modalités de préparation aux premières rencontres d'une classe, dans Jeunes enseignants et insertion professionnelle. Un processus de socialisation? De professionnalisation? De transformation?, sous la direction de J.-C. Hétu, M. Lavoie et S. Baillauquès. Paris, Bruxelles : De Boeck Université, pp. 139-160.

PERRENOUD, P. (1993). Formation initiale des maîtres et professionnalisation du métier. Revue des sciences de l'éducation, vol. XIX, nº 1, pp. 59-76.

SAINSAULIEU, R. (1988). L'identité au travail. Les effets culturels de l'organisation. Paris : Presses de la Fondation nationale des Sciences politiques.

TARDIF, M., LESSARD, C. (1999). Le travail enseignant au quotidien. Contribution à l'étude du travail dans les métiers et les professions d'interactions humaines. Laval : Les Presses de l’Université Laval.

VAN ZANTEN, A. (2001). L'influence des normes d'établissement dans la socialisation professionnelle des enseignants : le cas des professeurs des collèges périphériques français. Éducation et francophonie, vol. XXIX, nº 1 . 